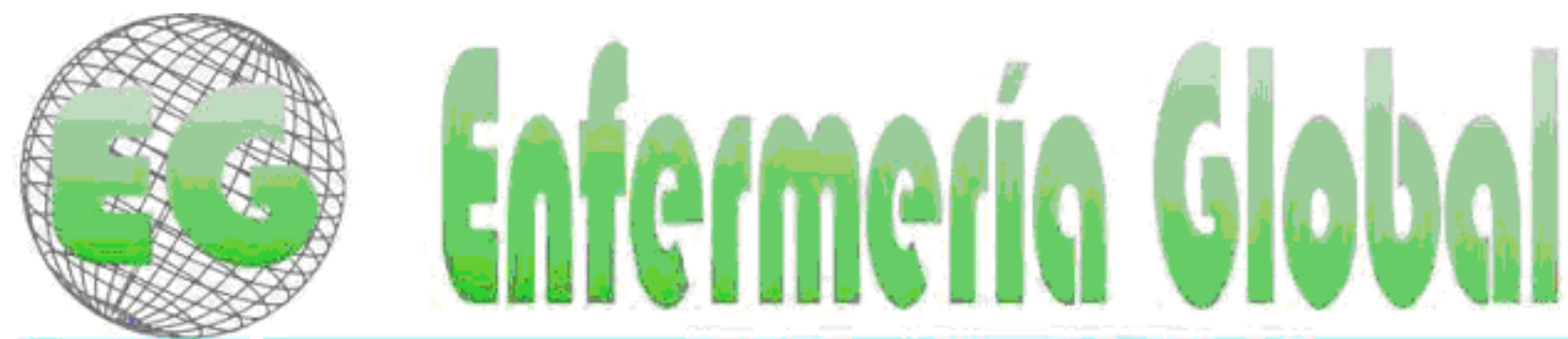

15SN $1696-6+59$

Revista electrônica cuatrimestral de Enfermeria

$\mathrm{N}^{\circ} 18$

Febrero 2010

www.um.es/egloball

\title{
MISCELÁNEA
}

\section{ACTUACIÓN DE LA ENFERMERÍA EN LA CREACIÓN DEL SERVICIO DE LACTANCIA DEL HOSPITAL JESÚS (1935-1938)}

A ATUAÇÃO DA ENFERMAGEM NA CRIAÇÃO DO LACTÁRIO NO HOSPITAL JESUS (1935-1938)

\begin{abstract}
${ }^{*}$ Rodrigues Pimenta, A., "*Moreira, A., ${ }^{* * *}$ Neves de Sant' Anna Menezes, A., ****Porto, FR., "Braga de Santana, S.

*Aluno do $8^{\circ}$ Período Curso de Graduação em Enfermagem da Universidade Estácio de Sá Campus Santa Cruz. **Doutora em Enfermagem Professora Associada do Departamento de Enfermagem Médico-Cirúrgica UNU-RIO/EEAP. ***Mestranda do Programa de Pósgraduação da UNIRIO- Mestrado em Enfermagem. Docente da Universidade Estácio de Sá. **** Doutor em Enfermagem. Professor Adjunto do Departamento Materno Infantil UNIRIO/EEAP. Brasil.
\end{abstract}

Palabras clave: Historia de la Enfermería, Nutrición

Palavras chave: Historia da Enfermagem, Nutrição.

Keywords: Nursing History, Nutrition.

\section{RESUMEN}

El presente estudio tiene como objeto la implantación del servicio de lactario en el Hospital Jesús y tiene por delimitación temporal el periodo de 1935-1938, que se justifica por la primera gestión del Servicio de Enfermería del Hospital Jesús, actual Hospital Municipal Jesús. La alimentación del niño merece cuidados especiales, principalmente en el primer año de vida. Objetivos: describir las políticas de alimentación en el Distrito Federal y analizar las circunstancias para la implantación del servicio de lactario en el hospital. Metodología: estudio con abordaje histórico social basado en el análisis documental, donde fueron utilizadas fuentes primarias y secundarias. Fuentes primarias: documentos escritos localizados en las dependencias del Hospital. Fuentes secundarias están constituidas de libros, disertaciones y trabajos referentes al desarrollo histórico del lactario. En el periodo abordado era necesaria la instalación de innumerables servicios dietéticos debidamente aparejados. Había la necesidad de un programa social y filantrópico, que rellenara las lagunas existentes en la protección al niño y fuera también órgano consultivo de los poderes públicos 
federales, estaduales y municipales. Fueron organizadas instituciones para asistencia a las madres y sus hijos. Juntamente a esta iniciativa, hubo mayor actuación del Estado en cuanto a la inspección médica de las escuelas y a la legislación del trabajo, para las madres y niños. Conclusión: A través de los documentos encontrados, se pudo observar con este estudio, la relevancia de la influencia del Servicio de Enfermería en la implantación del servicio de lactario del Hospital Jesús, teniendo en cuenta que el equipo responsable de la apertura, organización y manutención del lactario en la referida institución se constituyó en su mayoría de enfermeros, que actuaron desde la apertura del Hospital dando orientaciones en cuanto a la alimentación de los lactantes, que allí eran llevados en búsqueda de asistencia.

\section{RESUMO}

O presente estudo tem como objeto a implantação do serviço de lactário no Hospital Jesus e tem por delimitação temporal o período de 1935-1938, que se justifica pela primeira gestão do Serviço de Enfermagem neste Hospital, atual Hospital Municipal Jesus. A alimentação da criança merecia cuidados especiais, principalmente no primeiro ano de vida. Era elevado o numero de moléstias advindas da alimentação incorreta. Objetivos: descrever as políticas de alimentação no Distrito Federal e analisar as circunstâncias para implantação do serviço de lactário no hospital. Metodologia: estudo com abordagem histórico social embasado na análise documental, onde foram utilizadas fontes primárias e secundárias. Fontes primárias: documentos escritos, localizados nas dependências do Hospital. Fontes secundárias constituídas por livros, dissertações e trabalhos referentes ao desenvolvimento histórico do lactário. No período abordado era necessária a instalação de inúmeros serviços dietéticos. Existia necessidade de um programa social e filantrópico, que preenchesse as lacunas existentes na proteção à criança e fosse também órgão consultivo dos poderes públicos federais, estaduais e municipais. Foram organizadas instituições para assistência às mães e seus filhos. Juntamente com essa iniciativa, começou a existir maior atuação do Estado quanto, à inspeção médica das escolas e à legislação do trabalho, para mães e crianças. Essa conduta estabelecia uma divisão do trabalho, com uma predominância da ação privada no campo da assistência social. Conclusão: Por meio dos documentos encontrados, foi possível observar com este estudo, a relevância da influência do Serviço de Enfermagem na implantação do serviço de lactário do Hospital Jesus, tendo em vista que a equipe responsável pela abertura, organização e manutenção do lactário na referida instituição foi formada em sua maioria por enfermeiros, que atuaram desde a abertura do Hospital dando orientações no que se refere a alimentação dos lactentes, que ali eram levados em busca de assistência.

\section{INTRODUCCIÓN}

El objeto de este estudio es la implementación del servicio en el Hospital Lactário Jesús en la delimitación del período de 1935-1938, justificada por la primera gerencia del servicio de enfermería del hospital Jesús, actual Hospital Municipal Jesús de la Secretaría Municipal de Río de Janeiro. En el período de la delimitación temporal la institución fue dirigida por el doctor Alberto Borgeth, junto con la jefe del Departamento de Enfermería, enfermera Lucinda Silva de Araujo, egresada de la Escuela de Enfermeras Profesionales y Alfredo Pinto actual Escuela de Enfermería Alfredo Pinto, de la Universidad Federal del Estado de Río de Janeiro. El Hospital Jesús fue creado en 1935 durante la reforma de Pedro Ernesto en el Distrito Federal.

El Hospital Jesús tenía 150 camas para la atención de los niños. En este sentido, el lactario es una unidad obligatoria en todos los hospitales que tenían camas para niños y cunas para los recién nacidos. El lactario es para la preparación de leche y sustitutos, a partir de técnicas apropiadas con el fin de ofrecer la nutrición de los niños sin riesgo de contaminación.(Capasciutti,1977,455). 
Algunos criterios para el éxito del sector dependen de la localización y las cuestiones relacionadas con el hospital, tales como: adecuada previsión de espacio, distribución de áreas de trabajo; instalación del equipo necesario y una buena administración. El entendimiento en este sentido, señalizaba que el espacio previsto proporcionaba las técnicas de preparación de los alimentos y embotellado de la leche en botellas de manera satisfactoria. (Capasciutti, 1977,455).

Hacemos hincapié en que el desarrollo histórico de los lactários en el Brasil comenzó con el doctor Fernandes Figueira, director de la Escuela Profesional de Enfermeras y Enfermeros, en 1905-1906, dividida en la sección de la mujer con la Escuela de Enfermeras Profesionales Alfredo Pinto en 1920 (Rocha, 2008).

Este médico inició en Río de Janeiro el servicio de puericultura sistematizado cuando en 1909 asumió la dirección de la Policlínica de la Infancia, localizada en la calle Miguel Frías. En esta institución, la superficie de la planta baja del servicio de cuidado de los niños estaba prevista para las consultas de higiene infantil y la atención prenatal. Por otra parte, en esta misma superficie de la planta baja fue posible encontrar un modelo de cocina dietética. Desde esa cocina se distribuyó con una rigurosa higiene, los alimentos en sus diversas formas: leche, sopas y gachas. La creación de cocinas dietéticas fue uno de los más bellos logros del departamento de cuidado a los niños, apoyada por el entusiasmo del Dr Jesuíno de Albuquerque, que reconoció la necesidad y el valor práctico del hospital de alimentos (Ribeiro, 1943, 92 y 111).

Cuando fue creado el Hospital Jesús en 1935 la enfermería fue el engranaje principal para la implantación del servicio del lactario en la institución dada la necesidad de alimentar a los niños al carecer en aquella época de profesionales del área de nutrición.

A través de lo expuesto, el estudio tiene como objetivos: describir las políticas de poder en el Distrito Federal y examinar las circunstancias en el Hospital Jesús para la implantación del servicio del lactario y discutir la contribución de la enfermería en la alimentación infantil de la instituciñon.

\section{METODOLOGÍA}

Se trata de un estudio histórico social apoyado en análisis documental. Este análisis consistió en una serie de operaciones destinadas a estudiar y analizar los documentos para comprender la realidad social, política y económica con las cuales se relacionaran. En otras palabras, el análisis documental fue examinar los documentos, con el fin de investigar los factores sociales y sus relaciones con el tiempo socio-cultural-cronológico. (Richardson, 1999, 230)

Se utilizaron fuentes primarias y secundarias. Las fuentes primarias fueron documentos escritos ubicado en dependencias del Hospital Municipal Jesús, tales como: memorándums, las frecuencias y las escalas de los funcionarios, revistas del servicio público. Las fuentes secundarias estaban constituidas a partir de los libros de historia de Brasil, Revista Annaes de Enfermería de la Escuela de Enfermería Anna Nery y por las disertaciones y trabajos sobre el desarrollo histórico del lactario en las unidades de cuidado infantil y la adhesión al objeto de estudio.

\section{POLÍTICAS DE ALIMENTACIÓN EN EL DISTRITO FEDERAL}


La educación dietética se consideró de suma importancia, Margarida dos Passos Roza (1934), establece que la salud de los lactantes tenía una relación directa con la dieta, porque si el niño está bien alimentado el marco de la mortalidad infantil disminuía.

Por otra parte, también señala que la alimentación de los niños merecía especial atención, sobre todo en el primer año de vida, pues tasa de mortalidad era importante en esa etapa de vida del lactante. Fue elevado el número de enfermedades derivadas de la mala alimentación, llevando a la muerte al 50 por ciento de los niños menores de un año.

La educación de la población es el principal objetivo, el primer problema que debe resolverse por ser la base para la supervivencia de los niños, debido a que la población comparecía en las clínicas, pero sólo con la intención de lograr una prescripción. Para cambiar la actitud de la población era necesario instalar muchos servicios de alimentación debidamente equipados, con profesionales idóneos que pudiesen actuar en los puestos de higiene infantil y clínicas médicas, que buscan resultados para los enfermos y los niños sanos (Roza, 1934).

\section{LAS CIRCUNSTANCIAS PARA LA IMPLANTACIÓN DEL SERVICIO DE LACTÁRIO EM EL HOSPITAL JESÚS}

El médico Moncorvo Hijo en 1919, creó el Departamento de Niños, en Río de Janeiro, cuya responsabilidad de mantenimiento era del Estado, como era la intención de este médico, sin embargo, la agencia fue reconocida sólo como un servicio público en 1920. La institución existió hasta 1938 (Orlandi, 1985, p.84)

En 1934, la Inspección de Salud Infantil se convirtió en una Junta para la Protección de la Maternidad y la Infancia, por recomendación de la Conferencia para la Protección de la Infancia de Higiene, dada por el Dr. Gastão de Figueiredo - médico de la Inspección de Salud Infantil - en 1933, durante el gobierno de Getúlio Vargas.

Hubo necesidad de un programa social y filantrópico, que llenara las lagunas existentes en la protección del niño y también es un órgano asesor del gobierno federal, estatal y municipal (Fonseca, 1990, p.55).

De este modo, los médicos organizaron las instituciones con el objetivo de dar asistencia a las madres pobres y a sus hijos, teniendo en cuenta las necesidades relacionadas con la alimentación, el vestido y el asesoramiento higiénico. Junto con esta iniciativa, comenzó a exigir la responsabilidad del Estado en la inspección médica de las escuelas y la legislación laboral, para las madres y los niños. Esa conducta establecía una división del trabajo, con un predominio de la acción privada en el ámbito de la asistencia social (Pereira, 1992, p.13).

Con la Reforma Carlos Chagas en la década de los 20, tuvo lugar la reorganización de los servicios de salud pública, creándose el Departamento Nacional de Salud Pública (DNSP), que extendió sus operaciones a varios ámbitos, entre ellos la higiene de los niños. En este tiempo se fundó la Escuela de Enfermería de DNSP (más tarde la Escuela de Enfermería Anna Nery), de conformidad con el sistema Nightingale oriundo de Inglaterra y pasado de los Estados Unidos a Brasil.

Así, se institucionalizaba la ayuda al niño en el país, que a lo largo de cinco décadas (19201969), sufrió cambios en relación a las directrices de la protección de los niños. El servicio de Higiene Infantil del DNSP se transformó, lo que culminó en el movimiento del Departamento Nacional del Niño (DNCr) en 1940, cuyas responsabilidades se centraron 
fundamentalmente en acciones preventivas, mientras en el área curativa, se limitaba a la orientación y la organización así como a la supervisión de instituciones hospitalarias.

Cabe mencionar que el gobierno de Getúlio Vargas puso en marcha la construcción de hospitales de la Prefectura del Distrito Federal, entre ellos el Hospital Jesús que fue inaugurado en 20 de junio de 1935, considerado como una institución especializada en clínicas médicas y quirúrgicas de los niños (Departamento de Salud Estado de Guanabara, 1972, p.217-219).

El Hospital de Jesús fue construido después de un período de turbulencia política y económica en Brasil, que tuvo lugar después de la caída de la bolsa de valores de Nueva York y la crisis de gobierno en Brasil bajo la dirección de Pedro Ernesto, que consolidó su carrera profesional, destacando como dirigente por su trabajo en su clínica particular, siendo reconocido como un militante en los acontecimientos políticos de 1930. La visibilidad pública en la dirección de su casa de salud y la participación directa en la defensa militar de la Alianza Liberal fueron elementos que dieron a Pedro Ernesto confianza y legitimidad política y social para ser nombrado por Getúlio Vargas, entonces jefe del gobierno provisional instalado en octubre de ese año, para ejercerir el puesto de director nacional del Departamento de Asistencia Pública el 14 de noviembre de 1930. (Teixeira, 2004,25)

Los cuatro años en que estuvo en la alcaldía del Distrito Federal, como interventor, marcaron de manera indeleble su paso por el poder. En tanto que ejerció el cargo de interventor entre octubre de 1931 y abril de 1935, Pedro Ernesto puso en marcha una reforma en la asistencia municipal construyendo e inaugurando hospitales y dispensarios públicos municipales (Teixeira, 2004,31).

La apertura y funcionamiento del Hospital Jesús representó la concreción de la reforma, ya que el hospital fue construido e inaugurado en la administración de Pedro Ernesto. (Teixeira, 2004, 46)

\section{CONTRIBUCIÓN DE LA ENFERMERÍA DE ALIMENTACIÓN INFANTIL EN LA INSTITUCIÓN}

Los trastornos nutricionales son responsables, en alto porcentaje, por enfermedad de la primera infancia. Su causa principal es la comida, casi siempre manejada de manera irregular e inadecuada. Estando la salud del niño, especialmente de los lactantes, en relación directa con la alimentación dietética, así, alimentándose debidamente un niño, trabajaba para la disminución en el marco de la mortalidad infantil (Annaes de enfermería, año II, abril de 1934).

Según Machado (1949), la función del nutricionista es una de las principales tareas de enfermería en salud pública, principalmente en Brasil, donde el hambre y la malnutrición casi siempre han tenido tasas sorprendentes y alarmantes. Es la enfermera de la nutrición, la responsable de la aplicación, distribución y notas relativas a la alimentación, así como del peso periódico y estado nutricional de cada niño, y también para informar los cambios, tratando así de crear en el hospital la noción de la necesidad de una alimentación adecuada y la higiene adecuada. (Anales Enfermería Tomo II, apartado 4, octubre de 1949).

De esta manera, es importante señalar que con la creación del Hospital Jesús, para un buen funcionamiento, fue necesario establecer un lactario en la unidad, que era sólo para niños hospitalizados y matriculados en la clínica, que funcionaba de 7 a 15 horas y estaba 
subordinado directamente al Director del Hospital, bajo la responsabilidad de la enfermera dietista. (Libro de envío de compromiso, 1937, 362)

Hubo detrás de Hospital Jesús en el anexo del pabellón, una "gran lactario", con sala de aula para las madres, sala de confección, sala de distribución, sala de esterilización de las mamaderas, con estufa y sala de entrega. Había también una entrada para recepción y almacenamiento de material, y una entrada para dos ambulatorios. El Área destinada al lactário era amplia, pero con una planta poco funcional, pues había sido mal planeada por uno de los asesores del Director. El lactario poseía también un aparato para clorar el agua, que sólo estuvo en uso en cortas y peligrosas experiencias (Meira,1971,195).

El texto siguiente confirma lo expuesto por el autor, al referirse a la estructura inicial de construcción del Hospital Jesús.

"Es lamentable no haber sido el edificio del Hospital Jesús, construido bajo la dirección técnica adecuada desde su inicio, y sí, haber sido adaptación del edificio en construcción, pues de tal hecho resultó la exigüidad del esfuerzo, impropiedad de las divisiones internas, no adaptable al funcionamiento justo de diversos servicios."

(Informe del libro de los envíos de compromiso con la unidad, de fecha 13 de diciembre de 1937, pagina362)

Las clases para las madres no salieron adelante por la falta de motivación de alumnos y profesores interesados. Resultado: en un corto espacio de tiempo el lactario se convirtió en un depósito de material y vestuario para los funcionarios.

Por las normas del Hospital sólo podían ser admitidos los niños de más de 3 años, pero al final del primer año de funcionamiento del Hospital en 1936, al ser demasiado grande el número de niños atendidos, se creó una "sala de recién nacidos." (Meira, 1971)

Las dietas de los lactantes para los pabellones estaban dispuestas en la copa donde funcionaba el departamento de pediatría ya que el "gran lactario" estaba desactivado siendo este nuevo espacio de preparación de dietas, citado en su momento como "microlactario". (Meira, 1971).

La enfermería de lactantes dio prioridad al trabajo en equipo, al igual que en todas las áreas del Hospital Jesús. El tratamiento fue predominantemente dietoterápico, algo que se necesitaba en una unidad donde el $85 \%$ de los casos presentaba desnutrición crónica. Comienza así una nueva actuación en relación con el funcionamiento del lactario en la unidad, que tenía inicialmente siete funcionarios en el equipo perteneciente al departamento de enfermería y otras siete personas colocadas, citadas como "trabajadores" en el documento de unidad (Libro de la remesa del empeño de 11 de marzo de 1939), lo que en términos porcentuales significa un total del cincuenta por ciento del equipo formado por enfermería. (Libro en consignación de compromiso con la unidad 1937).

La responsabilidad del lactario recaía sobre la enfermera dietista - Irene Rocha da Silva que entre otras funciones controlaba la frecuencia de los niños que se beneficiaban con el lactario, administraba el material utilizado y realizaba reuniones con las madres de los niños que asistieron a la unidad, para el asesoramiento en materia de higiene infantil y la experiencia en la preparación de las dietas. 
Tomando como primer responsable a la enfermera Irene Rocha da Silva, formada por la Escuela de Enfermería Anna Nery. A través de este hecho se puede ver la importancia de la actuación de enfermería en la creación y desarrollo del lactario de esta institución.

\section{CONSIDERACIONES FINALES}

La alimentación era una de las principales preocupaciones para la salud del bebé, algo esencial para la vida, principalmente en el primer año, ya que era factor decisivo para la salud y el bienestar, pues en la década de 1930 la tasa de mortalidad infantil era significativa en esa etapa de vida del lactante. Era elevado el número de enfermedades derivadas de la alimentación incorrecta, conduciendo a la muerte al cincuenta por ciento de los niños menores de un año. (Anales de Enfermería, enero., 1935, 24, Roza, 1934).

Sumergido en un escenario de la salud de los niños nada prometedor, el Hospital Jesús fue construido después de un período de turbulencia política y económica en Brasil, con ocurrencia después de la caída de la bolsa de valores de Nueva York y la crisis de gobierno en Brasil, bajo la dirección de Getúlio Vargas como presidente, Pedro Ernesto Batista como Interventor del Distrito Federal. Durante un período de inestabilidad presente a principios de esta institución, la enfermería fue el principal engranaje para implantar el servicio de lactario en la institución de manera eficaz, debido a la necesidad de nutrición de los niños por carecer en este momento de profesionales en el ámbito de la nutrición. La responsable del lactario era la enfermera dietista y los profesionales en el área de enfermería se mantuvieron en la composición de un porcentaje importante del equipo del lactario hasta el extremo de la gestión de Lucinda de Araujo Silva.

Por los documentos encontrados, el lactario en el Hospital Jesús fue creado de manera exitosa en el año 1937, y su equipo de trabajo tenía papel importante en la concreción y la consolidación de este servicio que en su inicio tuvo muchas dificultades.

Basándose en lo anterior, es posible decir que el departamento de enfermería fue la base para la implantación eficaz del lactario en el Hospital Jesús. Donde los enfermeros, habían actuado de manera práctica al final del primer año de apertura de la unidad, dando también, las directrices con respecto a la nutrición de los lactantes, que fueron traídos en busca de ayuda.

\section{REFERENCIAS BIBLIOGRÁFICAS}

1. Amorim WM. A reconfiguração da primeira escola de enfermagem brasileira: a missão de Maria de Castro Pamphiro, 1937-1949. [tese] Rio de Janeiro (RJ): Escola de Enfermagem Anna Nery, Universidade Federal do Rio de Janeiro; 2004.

2. Capasciutti AS, et al. Planejamento de um lactário para um hospital escola de 400 leitos. Rev Saúde Públ São Paulo 1977; 11:455-64.

3. Fausto B. Historia do Brasil. Universidade de São Paulo/Fundação do Desenvolvimento da Educação, 2ª ed. São Paulo; 1995. p. 318-351.

4. Fonseca CMO. Modelando a cera virgem: a saúde da criança na política social de Vargas. [dissertação] Niterói (RJ): Instituto de Ciências Humanas e Filosofia da Universidade Federal Fluminense; 1990. 
5. Machado MCM. Educação em ambulatório de pediatria. Rev Annaes de Enfermagem 1949 volume II, $\mathrm{n}^{\circ} 4,1949$ out).

6. Oliveira MAC, Egry EY. A historicidade das teorias interpretativas do processo saúdedoença. Rev Esc Enferm USP 2000; 34:9-15.

7. Orlandi OV. Teoria e Prática do Amor a Criança: introdução à pediatria social no Brasil. Rio de Janeiro: Zahar; 1985.

8. Pereira ARVV. Políticas Sociais e Corporativismo no Brasil: O Departamento Nacional da Criança no Estado Novo. [dissertação] Niterói (RJ): Instituto de Ciências Humanas e Filosofia da Universidade Federal Fluminense; 1992.

9. Richardson RJ. Pesquisa social: métodos e técnicas. São Paulo: Atlas; 1999.

10. Ribeiro AM. A Defesa da Criança no Brasil. Rev do Servidor Público Rio de Janeiro. Ano VI, Vol. $1 \mathrm{n}^{\circ} 03$, março de 1943.

11. Rocha B, Moreira A, Porto F. Primeiros Passos de Antônio Fernandes Figueira como diretor da Escola Profissional de Enfermeiros e Enfermeiras. In: 7ạ Jornada de Iniciação Científica da UNIRIO, 2008. Livro de resumos. Rio de Janeiro, 2008. v. 1.

12. Roza MP. Serviço de Dietética Infantil. In: Anais de Enfermagem; 1934 abr. ano II; p. 24.

13. Secretaria de Saúde do Estado da Guanabara. Assistência Pública - Guanabara - 80 anos de Historia. Rio de Janeiro (RJ); 1972.

14. Teixeira CRRR. A Reforma Pedro Ernesto (1933): Perdas e Ganhos para os Médicos do Distrito Federal. [tese] Rio de Janeiro (RJ): Escola de História das Ciências da Saúde da Fundação Oswaldo Cruz; 2004.

15. Da Mortalidade Infantil e suas Causas. In: Annaes de Enfermagem; 1935 jan. $n^{\circ} 6$, vol. 07

16. Hospital Jesus. Livro de Remessa de Empenho (1938-1939) - Relatório; 1937 dez. Rio de Janeiro (RJ); 1937. p. 362-363.

17. Hospital Jesus. Livro de Remessa de Empenho (1938-1939) - Ante Projeto do Quadro de Pessoal de Enfermagem; 1937 nov. Rio de Janeiro (RJ); 1937.

18. Hospital Jesus. Livro de Ofícios Recebidos da Diretoria de Higiene e Assistência Hospitalar - Regimento do lactario. Rio de Janeiro (RJ); 1938.

19. Hospital Jesus. Livro de Remessa de Empenho (1938-1939) - Escala de Serviço; 1939 mar. Rio de Janeiro (RJ); 1939.

ISSN 1695-6141

๑ COPYRIGHT Servicio de Publicaciones - Universidad de Murcia 